\title{
Phytotherapie bei obstruktiven Atemwegserkrankungen
}

\author{
Autor: Peter W. Gündling
}

\section{ZUSAMMENFASSUNG}

Obgleich Phytotherapeutika bei Asthma und COPD nicht an erster Stelle stehen, weisen sie doch Wirkungen auf, die sowohl für das akute als auch das chronische entzündliche Geschehen obstruktiver Atemwegserkrankungen eine wichtige Rolle spielen: Schutz vor Infekten, antiinflammatorische und antivirale Wirkung, Verbesserung der mukoziliären Clearance, antitussive Wirkung, Immunmodulation, Schutz vor bakterieller Superinfektion.

Bei Beachtung der Grenzen dieser Therapierichtung stehen dem Therapeuten in der Praxis bewährte und wirksame Optionen zur Verfügung.

\section{Schlüsselwörter}

Phytotherapie, COPD, Asthma, obstruktive

Atemwegserkrankung

\section{ABSTRACT}

Even though phytotherapeutic agents are not the first choice for the treatment of asthma and COPD, they show effects, which are important for the acute and chronic inflammatory process of obstructive respiratory diseases: protection against infections, anti-inflammatory and antiviral effect, improvement of the mucociliary clearance, antitussive effect, immunomodulation, protection against a bacterial superinfection.

When respecting the limits of this form of therapy, effective options which have their proven worth in practice are available to the therapist.

Keywords

Phytotherapy, COPD, asthma, obstructive respiratory disease

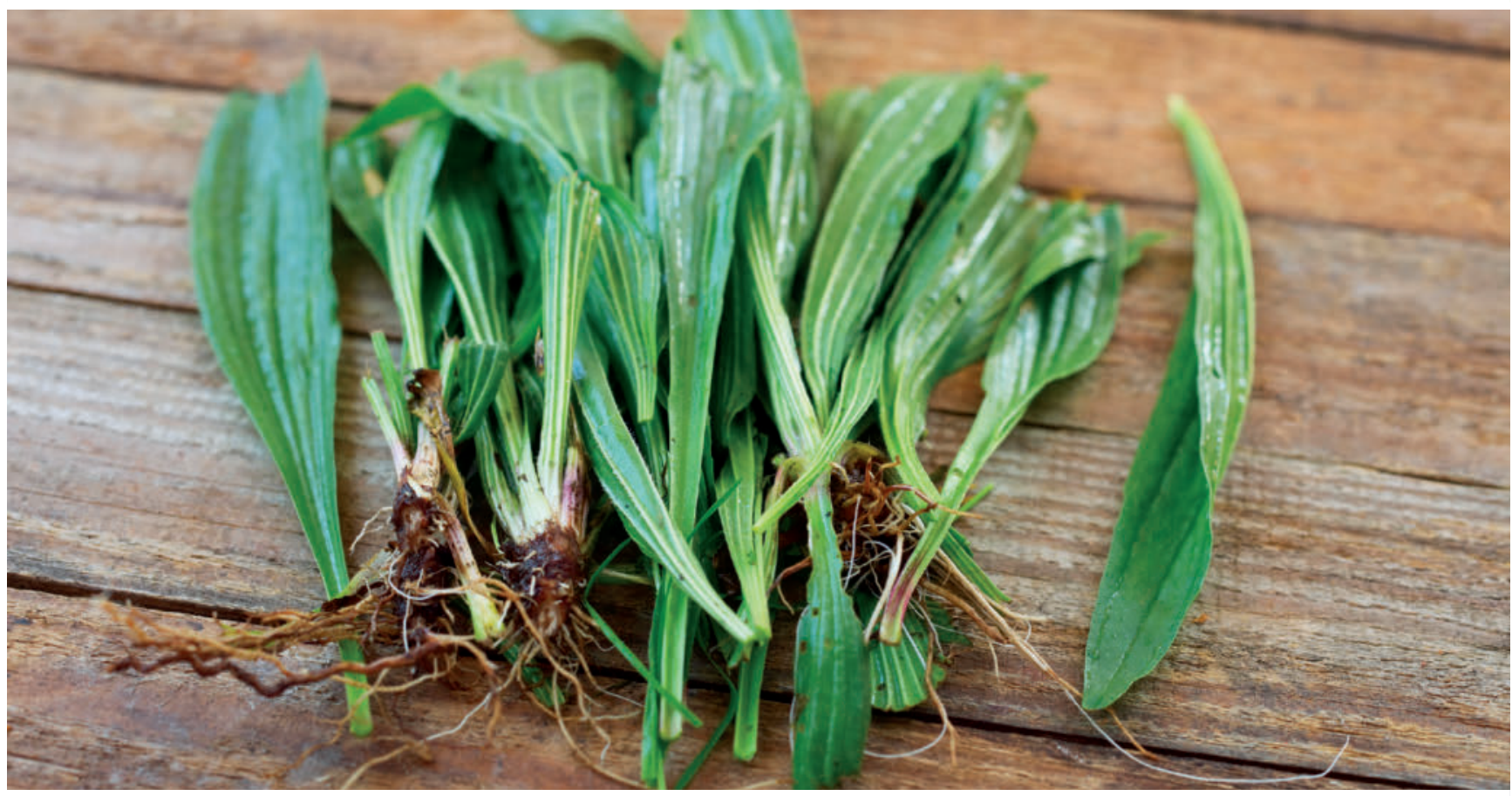

Abb. 1 Spitzwegerichkraut (Plantaginis lanceolatae herba) - positiv monographierte Schleimdroge bei schmerzhaftem trockenen Husten. (c) rainbow33/Adobe Stock

In jüngster Zeit, speziell in den letzten beiden Jahren, hat sich in der Pharmakotherapie der chronisch obstruktiven Atemwegserkrankungen sehr viel getan. Im Zuge der neuen Behandlungsempfehlungen des „Global Initiative for Chronic Obstructive Lung Disease“(GOLD)-Konsortiums werden nun sowohl die Stadieneinteilung als auch die daraus folgende Therapie praktisch ausschließlich von den Symptomen, der Leistungsfähigkeit und Lebensqualität sowie dem Risiko von Exazerbationen von COPD-Patienten bestimmt [1]. Diese seit 2017 geltenden Empfeh- lungen sind international maßgebend und stellen nun die Grundlage nationaler Leitlinien dar.

Pharmakologische Standardtherapie der COPD ist nun die Kombination von LAMA (= Long Acting Muscarin Antgonists, langwirksame Anticholinergika) und LABA (= Long Acting Beta Agonists, langwirksame Betamimetika), die eine duale Bronchodilatation bewirken und in Studien die beste Symptom- und Exazerbationskontrolle zeigen [2]. 
Damit ist der Einsatz von Glukokortikoiden bei diesen Patienten weit nach hinten gerückt und wird nur noch in schwersten Fällen (GOLD IV) und bei Patienten, die gleichzeitig unter Asthma bronchiale leiden, angewandt. Beim Asthma bronchiale ist es dagegen nach wie vor die Kombination aus Betamimetika und inhalativen Glukokortikoiden, die den leitliniengerechten Standard bestimmen. Pflanzliche Arzneimittel finden sich weder in den Leitlinien zur Therapie der COPD noch zum Asthma bronchiale.

Um also den Einsatz von Phytopharmaka zu rechtfertigen, braucht es den Blick auf die Begleitumstände, auf den speziellen Fall, auf das Individuum. Patienten, die trotz leitliniengerechter Therapie keine ausreichend gute Symptomkontrolle haben, Patienten, die die Leitlinienmedikation nicht vertragen oder bei denen diese nicht oder noch nicht indiziert ist. Das sind z. B. Kinder, multimorbide Patienten mit Hypertonie und/oder Herzinsuffizienz, für die Betamimetika nicht so ohne Weiteres zuträglich sind, oder auch das Stadium GOLD I.

Trotz der relativ kleinen Effektstärken punkten Phytotherapeutika durch ihr breites Wirk- und Anwendungsspektrum. Sie weisen Wirkungen auf, die weder LAMA noch LABA haben, die aber dennoch für das chronisch entzündliche Geschehen eine wichtige Rolle spielen: Schutz vor Infekten, antiinflammatorische und antivirale Wirkung, Verbesserung der mukoziliären Clearance, antitussive Wirkung, Immunmodulation, Schutz vor bakterieller Superinfektion. Zudem sind sie sehr gut verträglich, haben ein sehr geringes Interaktionspotential und erfreuen sich deshalb einer hohen Akzeptanz und Therapietreue bei den Betroffen.

\section{Akute Atemwegsinfekte als Auslöser obstruktiver Atemwegserkrankungen}

Sowohl bei Kindern als auch bei Erwachsenen kommt es nicht selten bei akuten Atemwegsinfektionen zur bronchialen Obstruktion. Speziell bei Kindern ist diese akute Erkrankung vom chronischen Asthma bronchiale zu unterscheiden und wird oft als „spastische Bronchitis“ oder „Infektasthma“ bezeichnet. Solche obstruktiven Episoden mit exspiratorischer Dyspnoe, Giemen, Pfeifen und Brummen werden bei nahezu der Hälfte aller Säuglinge und Kleinkinder beobachtet [3]. Und meist sind es Virusinfektionen, die zu dieser infektgetriggerten Bronchialverengung führen. Betamimentika oder inhalative Kortikoide sind hierbei nur in den seltensten Fällen angezeigt. Sehr bewährt haben sich dagegen Phytopharmaka, einerseits als frühe Virustatika, anderseits zur Entzündungshemmung und Bronchospasmolyse.

Doch diese Infekte machen nicht nur vorübergehende obstruktive Symptome, sie sind auch Wegbereiter für eine Asthmaentstehung und Trigger der Asthma- und COPD-
Exazerbation. Ersteres vor allem bei Kindern, letzteres bei Erwachsenen. Deshalb gilt es, akute Atemwegsinfekte frühzeitig zu bekämpfen und der Exazerbation die Grundlage zu nehmen.

\section{Graubehaarte Zistrose}

Sehr gut wirksam gegen akute virale Atemwegsinfekte vor allem im frühen Stadium ist der polyphenolreiche Extrakt aus der graubehaarten Zistrose (Cistus incanus). Dieses Phytotherapeutikum (Cystus 052 oder Cystus Pandalis ${ }^{\circledR}$ ) ist in der Lage, auf physikalischem, also nicht pharmakologischem Wege, das Eindringen von Viren in Schleimhautzellen zu verhindern und damit sowohl vor Erkältungs- als auch vor Grippeviren wirksam zu schützen [4][5]. Um das Eindringen von Viren zu verhindern, muss der Extrakt jedoch möglichst frühzeitig, also am besten vor oder ganz zu Beginn einer Virusinfektion eingenommen werden. Bei beginnenden Halsschmerzen, Husten oder Schnupfen beispielsweise alle 2-3 Stunden 1-2 Halspastillen [6]. Bei banalen Infekten und ganz früher Einnahme genügt häufig eine einzige Dosis, um den Infekt zu stoppen.

\section{Kaplandpelargonie}

Sowohl gegen Viren als auch gegen Bakterien wirksam ist der Extrakt aus der Wurzel der Kaplandpelargonie (Pelargonium sidoides). Seine antivirale Wirkung resultiert einerseits aus der Steigerung der Interferonproduktion [7], andererseits aus der gesteigerten Freisetzung antimikrobieller Peptide (Defensine) [8]. Dadurch wirkt er direkt antiviral, schützt Zellen vor Viruszerstörung und aktiviert natürliche Killerzellen. Seine antibakterielle Wirkung ist Folge der Verhinderung der Adhäsion von Bakterien an Schleimhautzellen, der Verhinderung der Internalisierung von Bakterien in Schleimhautzellen [9] sowie der Steigerung der Phagozytose und des intrazellulären Killings der Bakterien [10]. Zudem fördert er die Sekretomotorik durch die Steigerung der Zilienschlagfrequenz [11] und führt somit alles in allem zu einer nachweislich beschleunigten Heilung einer Bronchitis [12]. Darüber hinaus fanden Tahan und Yaman Hinweise dafür, dass dieser Extrakt das Risiko für die Entwicklung eines Asthmas bei Kindern mit akuten Atemwegsinfekten verringern kann [13]. Dieser Extrakt ist als Fertigarzneimittel (Umckaloabo ${ }^{\circledR}$ ) in Form von Tropfen, Tabletten und Saft für Kinder ab einem Jahr erhältlich.

\section{Kapuzinerkresse und Meerrettich}

Vor allem antibakteriell, aber auch antiviral, und deshalb auch gut bei Atemwegsinfektionen einsetzbar, wirken Kapuzinerkresse (Tropaeolum majus) und Meerrettich (Armoracia rusticana), die als Kombination in dem Fertigarzneimittel Angocin ${ }^{\circledR}$ Antiinfekt enthalten sind. Hauptwirkstoffe sind die darin enthaltenen Senföle, deren antimikrobielle Wirkung gut belegt ist [14][15]. Je nach Schwere der Infektion können davon 2- bis 4-mal täglich 5 Filmtabletten eingenommen werden. In einer größeren randomisierten kontrollierten klinischen Studie konnte Fintelmann zeigen, dass diese Kombination nicht nur 


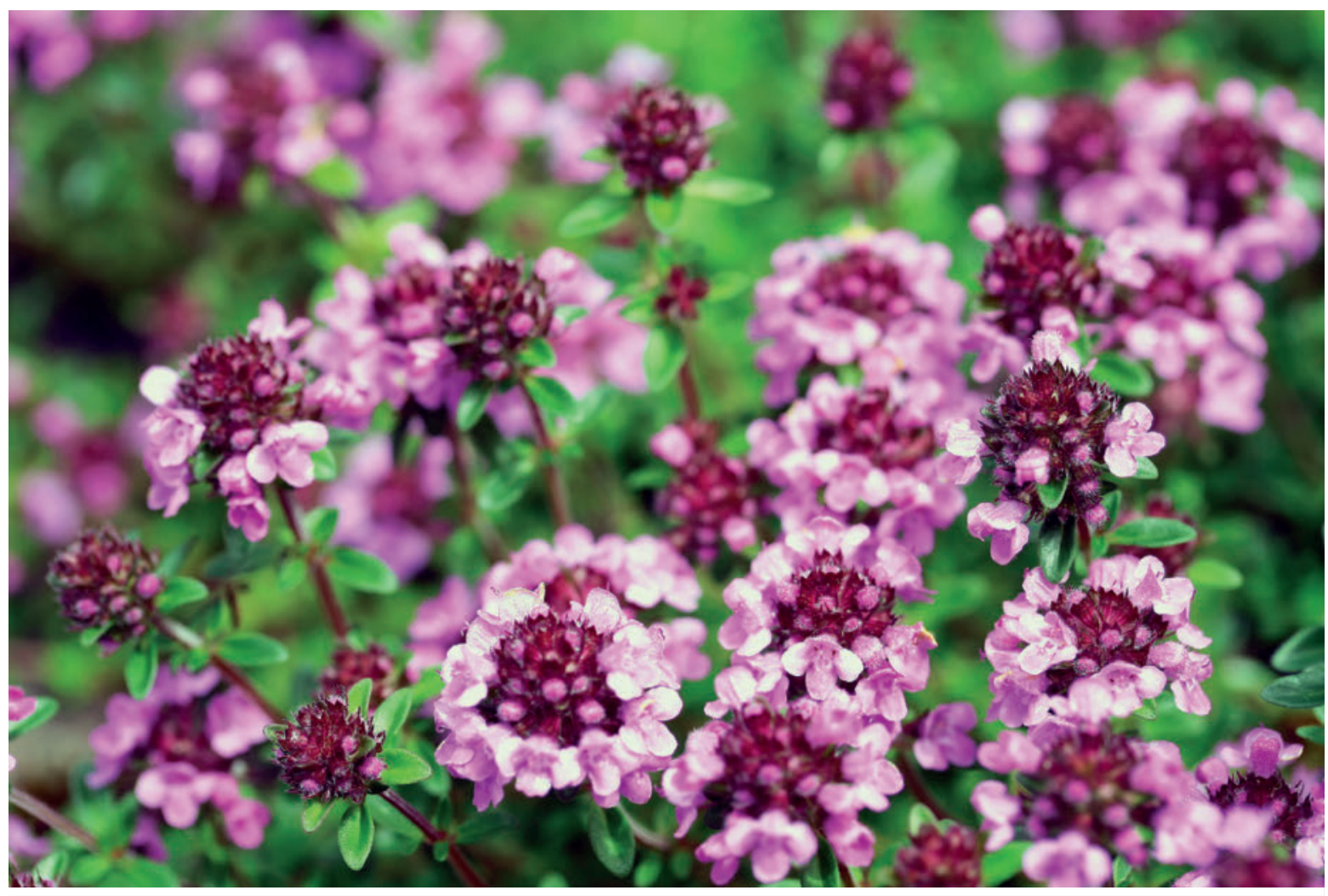

Abb. 2 Thymiankraut hat sich bei produktiver Bronchitis bewährt. @ Konstanze Gruber/Adobe Stock

therapeutisch wirksam ist, sondern auch prophylaktisch die Häufigkeit von Atemwegsinfektionen um 50\% reduziert [16].

\section{Roter Sonnenhut}

Der häufig eingesetzte rote Sonnenhut (Echinacea purpurea oder Echinacea angustifolia) ist in seiner antiviralen Wirkung etwas umstritten. Am besten wirkt er wohl prophylaktisch. Ist die Infektion bereits in vollem Gange, bringt er meist nicht mehr allzu viel. Hilfreiche Tees, die bei Erkältungskrankheiten das Immunsystem unterstützen und den Heilungsverlauf beschleunigen können, sind solche mit Hagebutte (Vitamin-C-reich) und/oder Lindenund Holunderblüten, die schweißtreibend und somit ausleitend wirken [6].

\section{Akute spastische Bronchitis, Infektasthma}

\section{Efeuextrakt}

Wie oben beschrieben, kommt diese Form der obstruktiven Atemwegserkrankung vor allem bei Kindern vor. Hier hat sich vor allem ein Extrakt aus den getrockneten Blättern des Efeus bewährt. Er enthält Saponine, Flavonoide, Phenolsäuren und ätherische Öle und weist neben antiinflammatorischen und mukolytischen Eigenschaften auch bronchospasmolytische auf [19]. Dass Efeu-Spezialextrakt (Prospan ${ }^{\circledR}$ ) zudem auch antitussiv wirkt, konnte Schaefer
2016 nachweisen: Bereits nach 48 Stunden reduzierte er die Hustenintensität signifikant im Vergleich zu Placebo. Nach siebentägiger Anwendung zeigte sich eine Reduktion um $69 \%$. Und das ohne unerwünschte Ereignisse oder Wechselwirkungen [20]. Da der Saft Kindern zudem sehr gut schmeckt, ist er bei spastischer Bronchitis in der Praxis oft erste Wahl.

Ebenfalls sehr bewährt bei diesem Krankheitsbild ist die Kombination von Efeu und Thymian, die z.B. in Bronchipret $^{\circledR}$ TE Saft und Tropfen enthalten ist. Diese Mischung kombiniert die brochospasmolytische und expektorierende Eigenschaft des Efeus mit den antimikrobiellen Eigenschaften des Thymians und kann daher - je nach vorherrschender Symptomatik - auch alternativ zu dem o.g. Monotherapeutikum eingesetzt werden.

\section{Bronchitis (produktiv)}

Bei der produktiven Bronchitis, wie sie häufig im Rahmen der COPD vorkommt, werden sog. Expektorantien eingesetzt, die sich in Sekretolytika ( $\rightarrow$ stimulieren die Bronchialsekretion), Sekretomotorika ( $\rightarrow$ stimulieren die mukoziliäre Clearance) und Mukolytika ( $\rightarrow$ verringern die Viskosität des Bronchialsekret) unterteilen lassen. Während chemisch definierte Wirkstoffe vorwiegend eine dieser Eigenschaften besitzen (z. B. Ambroxol = Sekretolytikum, Acetylcystein $=$ Mukolytikum), vermögen pflanzliche Mittel das Sekret zu verflüssigen, die Schlagfrequenz der Zi- 
lien zu steigern, den Sekretabtransport und das Abhusten (mukoziliäre Clearance) zu erleichtern, Entzündungen durch Hemmung proinflammatorischer Zytokine zu verringern und zudem antiviral und antibakteriell zu wirken. Rechtzeitig eingesetzt können sie damit eine bakterielle Superinfektion vermeiden und den Heilungsverlauf beschleunigen [6].

\section{Ätherischöldrogen und saponinhaltige Heil- pflanzendrogen}

Sekretolytisch wirkende Ätherischöl-Pflanzenextrakte sind Eukalyptusöl (Eucalypti aetheroleum), Fenchelöl (Foeniculi aetheroleum), Fichtennadelöl (Piceae aetheroleum) und Kiefernnadelöl (Pini aetheroleum). Sekretolytisch wirkende Blatt-, Kraut- und Früchtedrogen mit ätherischem Öl sind Anisfrüchte (Anisi fructus), Fenchelfrüchte (Foeniculi fructus), Salbeiblätter (Salviae folium) sowie Eukalyptusblätter (Eucalypti folium) und Thymiankraut (Thymi herba), die zusätzlich sekretomotorisch wirken.

Sehr bewährt und wirksam als Fertigarzneimittel in diesem Bereich ist eine Mischung der ätherischen Öle aus Eukalyptus, Süßorange, Myrte und Zitrone mit den Hauptwirkstoffen Myrtol, Cineol und a-Pinen. Diese Extraktkombination (ELOM-080) wirkt sekretolytisch und sekretomotorisch und steigert damit die mukoziliäre Clearance um 46\% [18]. Darüber hinaus wirkt sie antiinflammatorisch und bronchospasmolytisch. Sie ist in Form von Kapseln erhältlich (Gelomyrtol ${ }^{\circledR}$ forte) und für Kinder ab 6 Jahre zugelassen.

Cineol allein, der Hauptwirkstoff des Eukalyptusöls, gibt es ebenfalls als Fertigarzneimittel in Form von Kapseln (Soledum $^{\circledR}$ forte) und ist für Personen ab 12 Jahren zugelassen. Für dieses Phytopharmakon konnte nachgewiesen werden, dass es aufgrund seiner mukolytischen, bronchodilatorischen und antiinflammatorischen Eigenschaften als Begleitmedikation sowohl die Exazerbationsrate von COPDPatienten senken als auch die Krankheitssymptome und Lebensqualität von Asthmapatienten signifikant verbessern kann [21].

Die Dosierung besteht bei beiden Medikamenten aus dreibis viermal täglich einer Kapsel, am besten etwa $30 \mathrm{Mi}$ nuten vor einer Mahlzeit mit reichlich Flüssigkeit. Dann werden ätherische Öle am besten vertragen. Generell vorsichtig mit der Einnahme von ätherischen Ölen sollten allerdings magenempfindliche Personen sein sowie Patienten mit Erkrankungen im Bereich der Gallenwege und der Leber.

Neben diesen Ätherischöldrogen gibt es saponinhaltige Heilpflanzendrogen, die ebenfalls sekretolytisch expektorierend wirken. Unter Saponinen versteht man glykosidische Pflanzeninhaltsstoffe, die Detergens-Charakter (wie Seife) besitzen. Die Wirkung dieser Heilpflanzen beruht auf dem gastropulmonalen mukokinetischen Vagus- reflex, der für den schleimlösenden und auswurffördernden Effekt verantwortlich zeichnet [6].

Zu dieser Gruppe von Heilpflanzen gehören Efeublätter (Hederae helicis folium), Wollblume (Verbasci flos), Primelwurzel (Primulae radix), Rote Seifenwurzel (Saponariae rubrae radix), Weiße Seifenwurzel (Gypsophilae radix) und Senegawurzel (Polygalae radix). Wie bei seifenhaltigen Wirkstoffen nicht anders zu erwarten, können insbesondere die letztgenannten Wurzeldrogen zu Magenreizungen führen.

Sehr bewährte Medikamente dieser Gruppe sind vor allem Medikamente mit Thymian, Efeu und/oder Primel. Thymian als Monotherapeutikum ist z. B. in Thymipin ${ }^{\circledR}$ und Bronchicum ${ }^{\circledR}$ Lutschtabletten enthalten und hat außer einer stark sekretolytischen auch eine stark antiphlogistische, stark antibakterielle und antivirale Wirkung.

Die Kombination aus Efeublättern und Thymiankraut (Bronchipret ${ }^{\circledR}$ TE Saft oder Tropfen) wurde bereits oben beschrieben. Achtung: Im Unterschied zu dieser Flüssigarznei enthalten Bronchipret ${ }^{\circledR}$ TP Dragees ebenso wie Bronchicum ${ }^{\circledR}$ Saft, Tropfen und Kapseln eine Kombination aus Thymiankraut und Primelwurzel und wirken damit etwas anders. Ähnlich wie für Efeu konnte auch für diese Kombination nachgewiesen werden, dass sie Husten- und Bronchitissymptome signifikant schneller lindert als Placebo und dies bereits nach dem 2. Behandlungstag [22].

Während reine ätherische Öle vor allem in Form von Kapseln, Bronchialbalsam und Einreibungen Verwendung finden, können Blüten-, Blatt-, Kraut-, Früchte- und Wurzeldrogen nicht nur als Fertigarzneimittel in Hustensäften oder Tabletten, sondern auch als Tee verwendet werden. Ein bewährtes Rezept zum Selbermachen (s. - Kasten 1).

\section{KASTEN 1}

Arzneitee bei Bronchitis mit Verschleimung

$\begin{array}{ll}\text { - Spitzwegerichkraut } & 30.0 \\ \text { - Thymiankraut } & 15.0 \\ \text { - Schlüsselblumenwurzel } & 15.0 \\ \text { - Isländisch Moos } & 40.0\end{array}$

Abkochung: $1 \mathrm{EL}$ mit $500 \mathrm{ml}$ kaltem Wasser ansetzen, langsam aufkochen und 20 min kochen lassen, absieben, 2- bis 4-mal tägl. 1 Tasse trinken

\section{Laryngitis, Tracheitis, Reizhusten}

Besonders quälend und gleichzeitig recht schwer zu behandeln ist schmerzhafter trockener Husten. Hier hat die Phytotherapie sog. Schleimdrogen (Muzilaginosa) zu bieten. Das sind Heilpflanzen, die Mucopolysaccharide enthalten, die sich über die Schleimhäute von Pharynx, Larynx und Trachea legen, diese vor Irritationen schützen 
und damit hustenlindernd wirken. Postiv monographierte Pflanzendrogen sind Eibischwurzel (Althaeae radix), Eibischblätter (Althaeae folium), Huflattichblätter (Farfarae folium), Isländisches Moos (Lichen islandicus), Malvenblüten (Malvae flos), Malvenblätter (Malvae folium), Spitzwegerichkraut (Plantaginis lanceolatae herba) und Wollblumen (Verbasci flos). Ein bewährtes Arzneitee-Rezept ist in - Kasten 2 zu finden.

Als Fertigarzneimittel gibt es hier Hustensäfte aus Eibischwurzel (Phytohusti ${ }^{\circledR}$ ) und aus Spitzwegerichkraut (BronchoSern ${ }^{\circledR}$ ) sowie Lutschpastillen mit Isländischem $\operatorname{Moos}\left(\right.$ IslaMoos $\left.{ }^{\circledR}\right)$.

\section{KASTEN 2}

Arzneitee bei trockenem Reizhusten

- Spitzwegerichkraut

- Malvenblüten

- Wollblumenblüten

- Süßholzwurzel

- Fenchelfrüchte (aufgestoßen)

Zubereitung: 1 TL pro Tasse mit heißem Wasser übergießen, 5-10 min ziehen lassen, 2-3 Tassen pro Tag trinken.

\section{Grenzen der Phytotherapie}

Natürlich gibt es auch Grenzen für den Einsatz von Phytotherapeutika. So ist ein schwerkranker Patient mit Asthma oder COPD nicht unbedingt prädestiniert für eine (primär) phytotherapeutische Behandlung. Dennoch können diese auch adjuvant zu einer leitliniengerechten Standardtherapie sehr hilfreich sein. Beispielsweise zur Infektprophylaxe (Cystus, Pelargonienwurzel), Entzündungshemmung (Myrtol, Thymian), Schleimlösung (Efeu, Thymian, Primel) oder Hustenlinderung (Spitzwegerich, Eibisch).

Kontraindiziert bei Asthma bronchiale, bronchialer Hyperreagibilität und akuten Exazerbationen von COPD ist lediglich die Inhalation von ätherischen Ölen.

Die wichtigste Grenze dieser Arzneimittel jedoch ist die eigene Kenntnis und Sicherheit des Therapeuten. Ebenso wie niemand mal eben so eine Operation durchführen würde, die er einmal gesehen oder gelesen hat, sollte man auch nicht leichtfertig mit Arzneimitteln umgehen, die man nicht kennt [6]. 


\section{Interessenkonflikt}

Der Autor gibt an, dass kein Interessenkonflikt besteht.

Autor

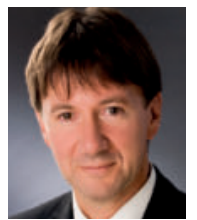

\section{Peter W. Gündling}

ist seit 1988 in eigener Praxis niedergelassen. Seit 2006 Professor für Naturheilkunde und komplementäre Medizin an der Hochschule Fresenius in Idstein, wo er als Studiendekan ein Masterstudium zur Naturheilkunde und komplementären Medizin entwickelt hat und leitet.

\section{Korrespondenzadresse}

Prof. Dr. med. Peter W. Gündling

Sebastian-Kneipp-Str. 4

65520 Bad Camberg/Ts.

E-Mail: guendling@hs-fresenius.de

\section{Literatur}

[1] Vogelmeier CF, Criner G], Martinez F] et al. Global Strategy for the diagnosis, management and prevention of chronic obstructive lung disease 2017 report. Respirology 2017; 22: 575-601

[2] Vogelmeier C, Buhl R, Burghuber O et al. Leitlinie zur Diagnostik und Therapie von Patienten mit chronisch obstruktiver Bronchitis und Lungenemphysem (COPD). [Guideline for the diagnosis and treatment of COPD patients - issued by the German Respiratory Society and the German Atemwegsliga in cooperation with the Austrian Society of Pneumology]. Pneumologie 2018; 72: 253-308

[3] Spindler T. Luftnot beim Kind. „Infektasthma“ oder Asthma bronchiale? Der Allgemeinarzt 2014; 36 (3): 18-22

[4] Ludwig S. Der Pflanzenextrakt Cystus052 blockiert Grippeviren. Zeitschrift für Phytotherapie 2012; 33: 14-18

[5] Kalus U, Grigorov A, Kadecki O et al. Cistus incanus (CYSTUS052) for treating patients with infection of the upper respiratory tract. A prospective, randomised, placebo-controlled clinical study. Antiviral Res 2009; 84: 267-271

[6] Gündling PW. Phytotherapie bei Atemwegserkrankungen. Die Naturheilkunde 2015; 92 (6): 18-21

[7] Kolodziej H. Fascinating metabolic pools of Pelargonium sidoides and Pelargonium reniforme, traditional and phytomedicinal sources of the herbal medicine Umckaloabo. Phytomedicine 2007; 14 (Suppl. 6): S9-17

[8] Koch E, Wohn C. Planta medica 2007; 73: 846: p072

[9] Conrad A, Jung I, Tioua D et al. Extract of Pelargonium sidoides (EPs 7630) inhibits the interactions of group A-streptococci and host epithelia in vitro. Phytomedicine 2007; 14 (Suppl. 6): S52-59

[10] Conrad A, Hansmann C, Engels I et al. Extract of Pelargonium sidoides (EPs 7630) improves phagocytosis, oxidative burst, and intracellular killing of human peripheral blood phagocytes in vitro. Phytomedicine 2007; 14 (Suppl. 6): 46-51

[11] Neugebauer $P$, Mickenhagen A, Siefer $O$ et al. A new approach to pharmacological effects on ciliary beat frequency in cell cultures - exemplary measurements under Pelargonium sidoides extract (EPs 7630). Phytomedicine 2005; 12: 46-51

[12] Chuchalin AG, Berman B, Lehmacher W. Treatment of acute bronchitis in adults with a pelargonium sidoides preparation (EPs 7630): a randomized, double-blind, placebo-controlled trial. Explore 2005; 1(6): 437-445

[13] Tahan F, Yaman M. Can the Pelargonium sidoides root extract $E P s \circledast 7630$ prevent asthma attacks during viral infections of the upper respiratory tract in children? Phytomedicine 2013; 20 (2): 148-150

[14] Conrad A, Biehler D, Nobis T et al. Broad spectrum antibacterial activity of a mixture of isothiocyanates from nasturtium (Tropaeoli majoris herba) and horseradish (Armoraciae rusticanae radix). Drug Res 2013; 63 (2): 65-68

[15] Kurepina N, Kreiswirth BN, Mustaev A. Growth-inhibitory activity of natural and synthetic isothiocyanates against representative human microbial pathogens. J Appl Microbiol 2013; 115 (4): 943-954

[16] Fintelmann V, Albrecht U, Schmitz G et al. Efficacy and safety of a combination herbal medicinal product containing Tropaeoli majoris herba and Armoraciae rusticanae radix for the prophylactic treatment of patients with respiratory tract diseases: a randomised, prospective, double-blind, placebocontrolled phase III trial. Curr Med Res Opin 2012; 28 (11): 1799-1807

[17] Bomblies I, Sonnenschein R. Antimicrobial action. In: Wittig T, ed. Myrtol standardized - A Clinical Documentation. Ergebnisse Verlag; 2005; 42-43

[18] Lai Y et al. Am J Rhinol Allergy 2014; 28 (3): 244-248

[19] Lang C, Röttger-Lüer P, Staiger C. A Valuable option for the treatment of respiratory diseases: review on the clinical evidence of the Ivy Leaves Dry Extract EA 575®. Planta Med 2015; 81: 968-974

[20] Schaefer A et al. Randomisierte, doppelblinde klinische Prüfung zur Wirksamkeit und Verträglichkeit des EfeuSpezial-Extraktes EA 575 bei der Behandlung von akutem Husten. Pharmazie 2016; 71(9): 505-510

[21] Worth H, Dethelfsen U. Patienten mit Asthma profitieren von einer Begleittherapie mit Cineol: eine placebokontrollierte Doppelblindstudie. Journal of Asthma 2005; 49(8): 849-853

[22] Schenk A. Kombination aus Thymiankraut und Primelwurzel zur Behandlung der Bronchitis eine Übersicht. Z Phytother 2017; 38: 117-120

\section{Bibliografie}

DOI https://doi.org/10.1055/a-0749-6465

EHK 2018; 67: 273-278

(c) MVS Medizinverlage Stuttgart GmbH \& Co. KG ISSN 0014-0082 